\title{
Исследование кристаллической и электронной структуры графеновых пленок, выращенных на $6 H$-SiC (0001)
}

\author{
() В.Ю. Давыдов ${ }^{1,3}$, Д.Ю. Усачёв ${ }^{2}$, С.П. Лебедев ${ }^{1}$, А.Н. Смирнов ${ }^{1,3}$, В.С. Левицкий ${ }^{1}$, И.А. Елисеев ${ }^{1,2}$, \\ П.А. Алексеев ${ }^{1}$, М.С. Дунаевский ${ }^{1}$, О.Ю. Вилков ${ }^{2}$, А.Г. Рыбкин ${ }^{2}$, А.А. Лебедев ${ }^{1}$
}

${ }^{1}$ Физико-технический институт им. А.Ф. Иоффре Российской академии наук, 194021 Санкт-Петербург, Россия

${ }^{2}$ Санкт-Петербургский государственный университет,

199034 Санкт-Петербург, Россия

${ }^{3}$ Университет ИТМО,

197101 Санкт-Петербург, Россия

E-mail: valery.davydov@mail.ioffe.ru

(Получена 31 января 2017 г. Принята к печати 8 февраля 2017 г.)

С использованием спектроскопии комбинационного рассеяния света, атомно-силовой микроскопии, дифракции медленных электронов, рентгеновской фотоэлектронной спектроскопии, фотоэлектронной спектроскопии с угловым разрешением и спектроскопии рентгеновского поглощения вблизи $K$-края углерода изучены структурные, химические и электронные свойства эпитаксиальных графеновых пленок, выращенных методом термического разложения $\mathrm{Si}$-грани полуизолирующей подложки $6 H$ - $\mathrm{SiC}$ в среде аргона. Показано, что результаты систематических комплексных исследований позволили оптимизировать ростовые параметры и создать надежную технологию роста высококачественных однослойных пленок графена с небольшой долей включений двухслойного графена.

DOI: 10.21883/FTP.2017.08.44800.8559

\section{1. Введение}

Графен (один монослой графита) в настоящее время является предметом обширных исследований, обусловленных его уникальными физическими свойствами и огромным потенциалом для создания устройств нового поколения, реализующих принципы баллистической электроники, спинтроники, оптоэлектроники, наноплазмоники и других перспективных альтернатив традиционной полупроводниковой электронике [1]. Для того чтобы быть экономически выгодными и привлекательными для многочисленных приложений, требуются большеразмерные пластины высококачественного однородного графена. Сублимация кремния из полуизолирующей монокристаллической подложки карбида кремния $(\mathrm{SiC})$ является одним из наиболее перспективных способов эпитаксиального роста графена [2,3]. Большим преимуществом данной технологии является отсутствие необходимости переносить выращенную пленку на подложку диэлектрика, как это имеет место, например, при синтезе графена на металлах. Кроме того, такая технология роста дает возможность выращивать графен на поверхности коммерческих подложек $\mathrm{SiC}$ диаметром до 4 дюймов, промышленный выпуск которых освоен в настоящее время. Подобные структуры могут быть использованы в стандартной технологической линейке для изготовления полупроводниковых приборов.

Структурные характеристики графеновой пленки существенно зависят от того, на какой грани $\mathrm{SiC}$ кристалла происходит рост $[2,4]$. Пленки графена, выращенного на С-грани, обычно состоят из нескольких разориентированных друг относительно друга монослоев, а топогра- фия их поверхности выглядит как отдельные чешуйки. В противоположность этому пленки, выращенные на Si-грани, имеют более однородную структуру поверхности, которая представляет собой длинные террасы шириной $\sim 1-2$ мкм. Как правило, такие пленки состоят из одного или совсем небольшого количества монослоев. Таким образом, морфологические и структурные характеристики графеновых пленок, выращенных на Si-грани $\mathrm{SiC}$, являются более благоприятными для создания приборных устройств на их основе.

Задачей настоящей работы являлась систематическая комплексная диагностика графеновых пленок, полученных методом термодеструкции $\mathrm{Si}$-грани подложки $6 H$-SiC, с целью установления связи их структурных, химических и электронных характеристик с технологическими параметрами роста.

\section{2. Образцы и эксперимент}

Для получения пленок графена использовался метод термического разложения поверхности $\mathrm{SiC}$. В отличие от работ $[5,6]$, где рост графена проходил в высоком вакууме, в данной работе использовалась усовершенствованная технология роста в среде инертного газа (аргона), позволяющая более точно контролировать процесс сублимации компонентов карбида кремния. Как следствие, с помощью подбора оптимальных технологических параметров можно с высокой точностью контролировать как процесс роста графеновой пленки, так и однородность покрытия подложки графеном.

В качестве подложки применялись высокоомные пластины $\mathrm{SiC}$ политипа $6 \mathrm{H}$ (производство ОАО „Светлана“) 
с ориентацией $(0001) \pm 0.25^{\circ}$ (Si-грань). Рост графена производился в графитовом тигле, нагреваемом индуктивным методом с помощью высокочастотного генератора. Для определения оптимальных условий получения графена осуществлялось варьирование основных технологических параметров: температура роста изменялась от 1750 до $1900^{\circ} \mathrm{C}$, время роста - от 10 до 30 мин, давление аргона в ростовой камере - от 600 до 800 Торр, скорость нагрева образца - от 100 до $350^{\circ} \mathrm{C} /$ мин.

Структурные, химические и электронные характеристики выращиваемого графена контролировались с использованием спектроскопии комбинационного рассеяния света (КРС), атомно-силовой микроскопии (АСМ), дифракции медленных электронов (ДМЭ), рентгеновской фотоэлектронной спектроскопии (РФЭС), фотоэлектронной спектроскопии с угловым разрешением (ФЭСУР) и спектроскопии рентгеновского поглощения вблизи $K$-края углерода (NEXAFS).

Измерения методом КРС проводились при комнатной температуре в геометрии „обратного рассеяния“ на спектрометрической установке LabRam HR 800, укомплектованной конфокальным микроскопом, что позволяло получать информацию из области графеновой пленки диаметром $\sim 1$ мкм. Наряду с локальной диагностикой, анализировались большие площади образцов размерами от $10 \times 10$ до $30 \times 30$ мкм с последующим построением КРС-карт параметров спектральных линий. Такая методика позволила реализовать одну из основных задач настоящей работы, которая заключалась в оценке степени однородности структурных характеристик по площади образцов. При измерениях использовалась длина волны возбуждающего света 532 нм; мощность лазерного излучения на образце равнялась $2.0 \mathrm{MBT}$ в пятне диаметром $\sim 1$ мкм; спектральное разрешение спектрометра составляло $2.5 \mathrm{~cm}^{-1}$.

Исследования топографии поверхности и распределения поверхностного потенциала производились с помощью сканирующего зондового микроскопа NtegraAURA (NT-MDT) в атмосферных условиях. Использовались полуконтактные зонды NSG11 (NT-MDT) c Pt проводящим покрытием. Измерения осуществлялись стандартной двухпроходной методикой, при этом в первом проходе регистрировалась топография поверхности. Во втором проходе, при поддержании расстояния между зондом и поверхностью в 20 нм, методом Кельвин-зонд микроскопии с амплитудной модуляцией осуществлялась регистрация поверхностного потенциала.

Данные ДМЭ, ФЭСУР и РФЭС с использованием рентгеновского излучения $\mathrm{Al} K_{\alpha}$ получены в Ресурсном Центре „Физические методы исследования поверхности“ Научного парка Санкт-Петербургского государственного университета (СПбГУ). Спектры РФЭС при различных энергиях фотонов, а также спектры NEXAFS измерены на оборудовании Российско-Германского канала вывода синхротронного излучения накопительного кольца BESSY II (г. Берлин).
Ниже приводятся результаты, которые являются типичными для образцов, выращенных на конечном этапе оптимизации технологических параметров.

\section{3. Экспериментальные результаты и их обсуждение}

\section{1. Данные КРС}

На рис. 1, $a$ представлен массив спектров КРС, измеренных в диапазоне $1300-2800 \mathrm{~cm}^{-1}$ на площади образца $12.5 \times 12.5$ мкм $^{2}$. В спектрах доминируют широкие полосы, обусловленные процессами второго порядка при рассеянии света от подложки $6 \mathrm{H}$-SiC. На рис. $1, b$ показан вид того же массива спектров, полученный после вычитания мешающего вклада спектра подложки. В спектрах наблюдаются особенности, возникающие при рассеянии света от графеновой пленки: линии $G, 2 D$ и слабая линия $D$ [7].

Линия $G$ в спектре графена соответствует нерезонансному рассеянию света с участием оптического фонона симметрии $E_{2 g}$ с малым волновым вектором. Этот
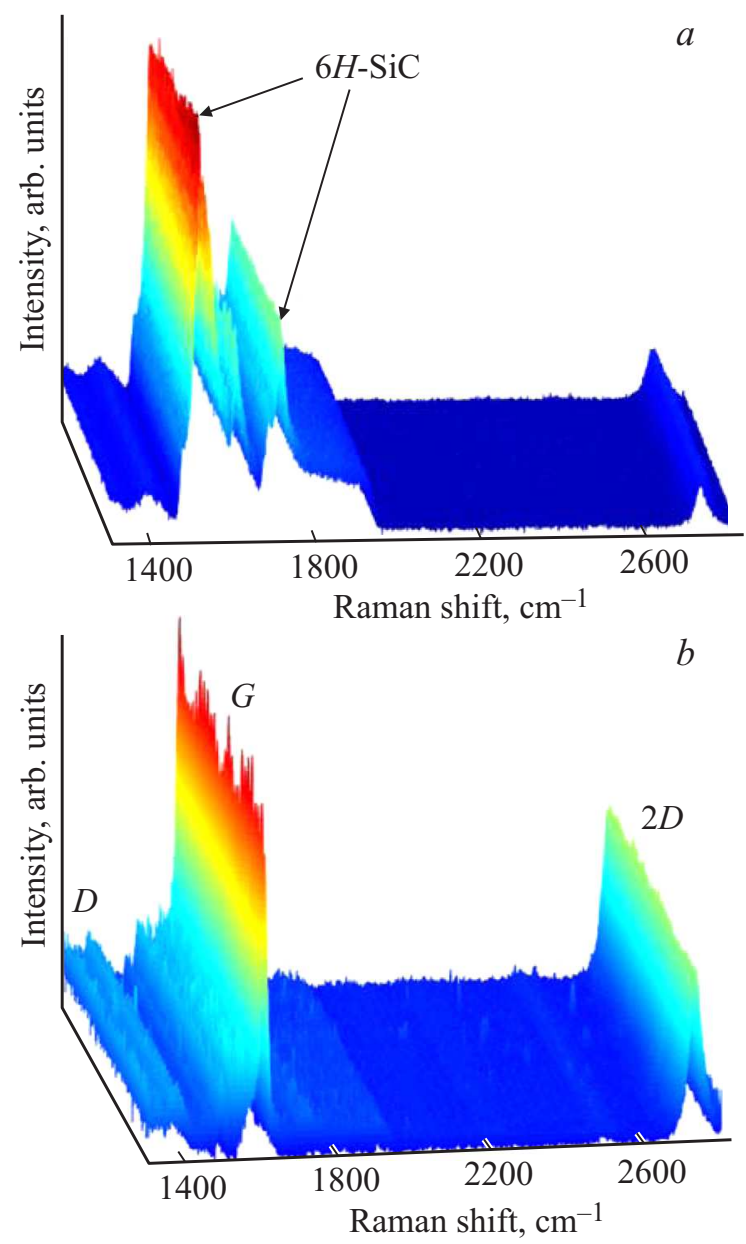

Рис. 1. Массив спектров КРС образца, выращенного на $\mathrm{Si}-г р а н и ~ 6 H-\mathrm{SiC}(a)$. Вид того же массива спектров, полученный после вычитания спектра подложки $(b)$. 
фонон вызван колебаниями атомов углерода в плоскости слоев. Появление линии $D$ в спектрах объясняется резонансным рассеянием с участием электронных состояний из двух неэквивалентных $K$ - и $K^{\prime}$-точек зоны Бриллюэна и оптического фонона с большим волновым вектором. Этот процесс запрещен правилом отбора по квазиимпульсу, однако условие его сохранения может быть выполнено, если в процессе рассеяния участвует также дефект кристаллической решетки. В структурносовершенных образцах графена линия $D$ не должна наблюдаться. Природа линии $2 D$, обертона линии $D$, также связана с резонансным рассеянием света с участием электронных состояний из $K$ - и $K^{\prime}$-точек зоны Бриллюэна, однако в этом процессе участвуют два оптических фонона с большими волновыми векторами. Они обладают одинаковой энергией, но противоположным направлением импульса. Условия сохранения квазиимпульса для такого процесса выполняются всегда, поэтому линия $2 D$ будет присутствовать в спектре графена, даже если в нем отсутствует линия $D$. Диагностика графеновых слоев с использованием данных спектроскопии КРС основана на анализе частотного положения, ширины и отношения интенсивностей $G, 2 D$ и $D$ линий в спектрах.

Карта распределения интенсивности линии $G$ по площади образца позволяет выявлять области, имеющие различную толщину; при этом областям с большим числом монослоев соответствует большая интенсивность сигнала. Анализ такой карты, полученной из обработки массива спектров, представленных на рис. $1, b$, выявил достаточно равномерное распределение интенсивности линии $G$ по площади образца. Это свидетельствует в пользу хорошей однородности толщины графеновой пленки в анализируемой области. Карта КРС интенсивности линии $G$ и результаты ее сравнительного анализа с данными АСМ приводятся в разделе 3.2.

Гистограмма карты значений полной ширины на половине высоты (ПШПВ, $F W H M$ ) линии $2 D$ представлена на рис. 2, $a$. Как следует из гистограммы (графика статистического распределения элементов карты), значения ПШПВ линии $2 D$ распределены в узком диапазоне величин с максимумом вблизи $33 \mathrm{~cm}^{-1}$. Было установлено, что форма линии $2 D$ в большинстве спектров имеет симметричный вид и хорошо аппроксимируется одиночным контуром Лоренца, что является признаком однослойного графена [7]. Форма линии 2D, которая может быть аппроксимирована огибающей четырех контуров Лоренца, наблюдается не более чем в $10 \%$ от общего числа спектров в массиве. Из этих данных следует, что анализируемый образец представляет собой преимущественно однослойный графен с небольшим количеством двухслойных включений.

Отношение интегральных интенсивностей $D$ и $G$ линий $\left(I_{D} / I_{G}\right)$ может быть использовано для оценки степени структурного совершенства графеновой пленки: чем меньше это значение, тем совершеннее пленка. На pис. $2, b$ приведена гистограмма карты отношений $I_{D} / I_{G}$. Видно, что максимум распределения отношения $I_{D} / I_{G}$
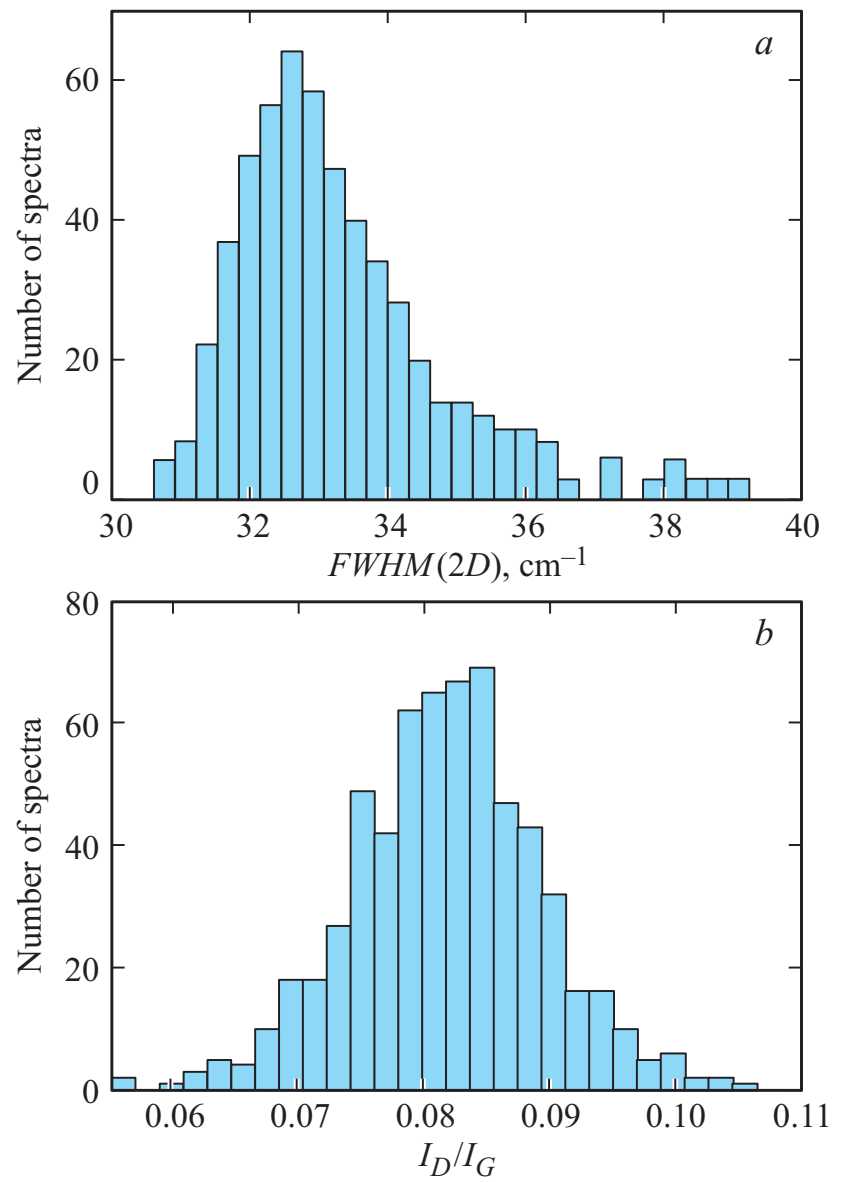

Рис. 2. Гистограмма карты КРС значений полной ширины на половине высоты (ПШПВ, $F W H M$ ) линии $2 D(a)$. Гистограмма карты КРС отношения интенсивностей $I_{D} / I_{G}(b)$.

находится вблизи 0.08 , что соответствует значению концентрации дефектов в кристаллической решетке $N_{d}<10^{10} \mathrm{~cm}^{-2}[8]$. Отношение $I_{D} / I_{G}$ может быть также использовано для оценки размера кристаллитов [9]:

$$
L_{a}=\left(2.4 \cdot 10^{-10}\right) \lambda_{l}^{4}\left(\frac{I_{D}}{I_{G}}\right)^{-1}
$$

здесь $L_{a}$ - размер кристаллитов в нм, а $\lambda_{l}=532 \mathrm{Hм}-$ длина волны возбуждающего излучения. С использованием формулы (1) и данных гистограммы, представленной на рис. $2, b$, оценка размера кристаллитов дает значения в диапазоне 190-320 нм.

Положение линии $G$ в спектрах образцов, выращенных на конечном этапе оптимизации, регистрируется на частотах имеющих большие значения, чем частота $\omega=1580 \mathrm{~cm}^{-1}$, характерная для объемного кристаллического графита. Наблюдаемый высокочастотный сдвиг можно объяснить двумя факторами: деформациями сжатия, которые возникают вследствие разницы коэффициентов термического расширения решетки графена и $\mathrm{SiC}$, и/или избытком заряда по сравнению с электрически нейтральным графеном [10-12]. 


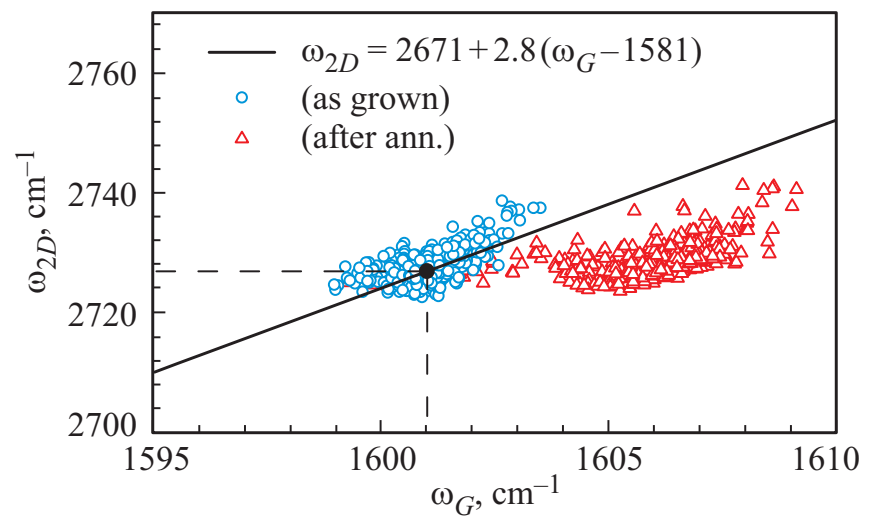

Рис. 3. Корреляция между положениями линий $2 D$ и $G$ (круглые символы - исходный образец, треугольные символы образец после его прогрева при температуре $500^{\circ} \mathrm{C}$ в сверхвысоком вакууме ). Сплошной линией показана зависимость (2), полученная для двуосно-деформированного электрически нейтрального графена в работе [14]. Пунктиром отмечены значения $\omega_{G}$ и $\omega_{2 D}$, используемые для оценки деформации $\varepsilon_{\|}$в образце.

Одним из способов, позволяющих разделить вклады деформации и избытка заряда, является совместный анализ положения линий $G$ и $2 D$ в предположении, что деформация является двуосной [13]. Важно отметить, что в отличие от линии $G$, положение линии $2 D$ в спектре зависит не только от деформации и избытка заряда, но также и от длины волны возбуждающего света. Это необходимо учитывать при сопоставлении экспериментальных данных, полученных в данной работе, с результатами, приводимыми в литературе. В работе [14] приводятся значения $\omega_{G}^{0}=1581 \mathrm{~cm}^{-1}$ и $\omega_{2 D}^{0}=2671 \mathrm{~cm}^{-1}$ для положений линий $G$ и $2 D$ в спектре КРС недеформированного электрически нейтрального графена. Эти значения были получены с использованием длины волны возбуждающего света 532 нм, которую мы также используем в данной работе. Кроме того, в работе [14] была установлена зависимость между положениями $G$ и $2 D$ линий для такого графена в условиях двуосной деформации:

$$
\omega_{2 D}=2671+2.8\left(\omega_{G}-1581\right) \mathrm{cm}^{-1} .
$$

На рис. 3 представлены данные о корреляции между положениями линий $2 D$ и $G$ для исходного образца, полученные в результате обработки соответствующих карт КРС. Хорошее согласие экспериментальных данных с зависимостью (2) указывает на то, что сильный высокочастотный сдвиг фононных линий, регистрируемый в образце, преимущественно связан с наличием деформации, имеющей характер сжатия. С использованием данных работы [14], величина деформации сжатия в плоскости слоя может быть оценена как $\varepsilon_{\|}=(0.35 \pm 0.03) \%$ из сдвига $\Delta \omega_{G}=20 \mathrm{~cm}^{-1}$, или как $\varepsilon_{\|}=(0.34 \pm 0.03) \%$ из сдвига $\Delta \omega_{2 D}=55 \mathrm{~cm}^{-1}$. Разброс величин для каждой оценки в пределах $10 \%$ говорит о наличии не очень большой локальной неоднородности в распределении деформации в образце.

На рис. 3 приводятся также значения частот линий $G$ и $2 D$, зарегистрированные в спектре после РФЭС измерений, которые были проведены в сверхвысоком вакууме и сопровождались предварительным прогревом образца при температуре $500^{\circ} \mathrm{C}$. Видно, что линия $G$ в спектрах испытывает дополнительный высокочастотный сдвиг. При этом диапазон положения линии $2 D$ остается практически неизменным. Такое поведение нельзя связать с возникновением дополнительной деформации в образце после прогрева, но его можно объяснить, если допустить появление избытка поверхностного заряда.

В работах $[11,12]$ было показано, что избыток поверхностного заряда, независимо от знака, всегда приводит к дополнительному высокочастотному сдвигу линии $G$. Линия $2 D$ реагирует по-разному на избыток поверхностного заряда, созданного электронами или дырками. Было установлено, что эта линия начинает сдвигаться в высокочастотную сторону даже при небольшой концентрации дырок. В то же время увеличение поверхностной концентрации электронов вплоть до $2 \cdot 10^{13} \mathrm{~cm}^{-2}$ не приводит к существенному изменению ее положения
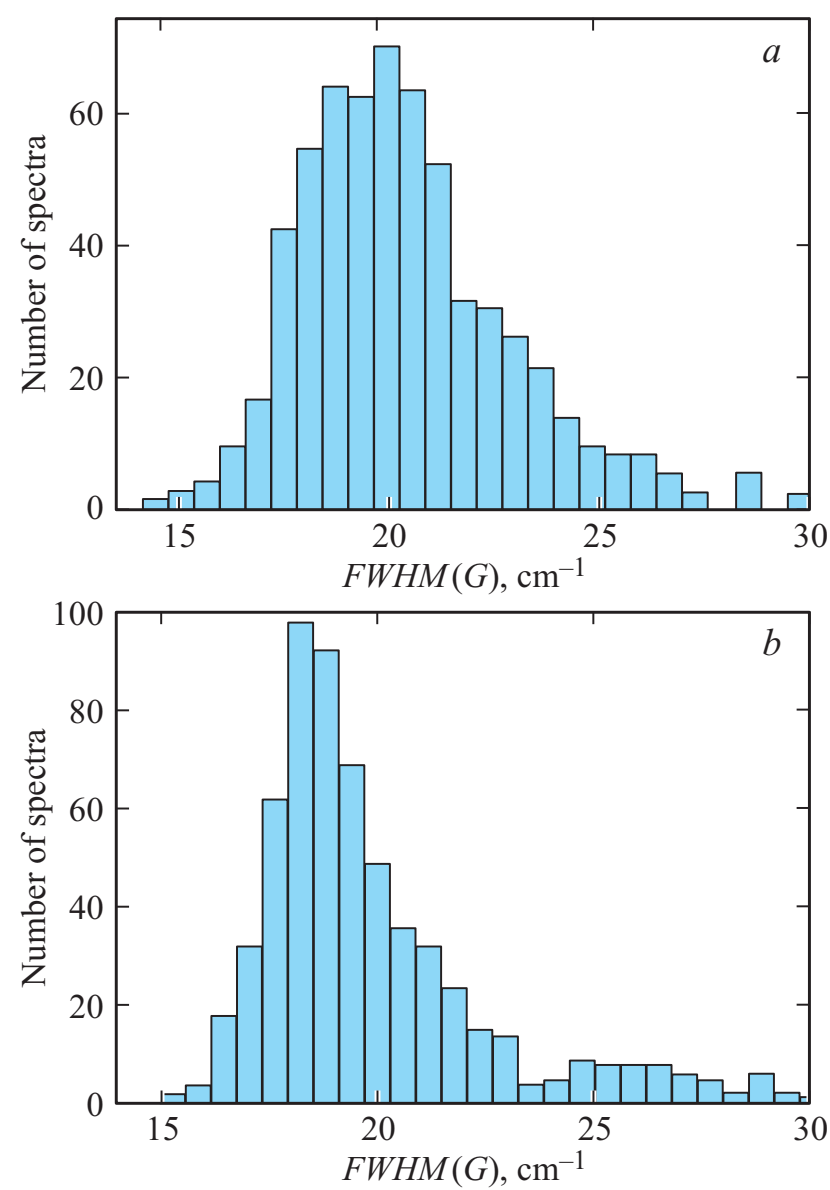

Рис. 4. Гистограмма карты КРС значений ПШПВ линии $G$ для исходного образца $(a)$. Гистограмма карты КРС значений ПШПВ линии $G$ для того же образца после его прогрева при температуре $500^{\circ} \mathrm{C}$ в сверхвысоком вакууме $(b)$. 


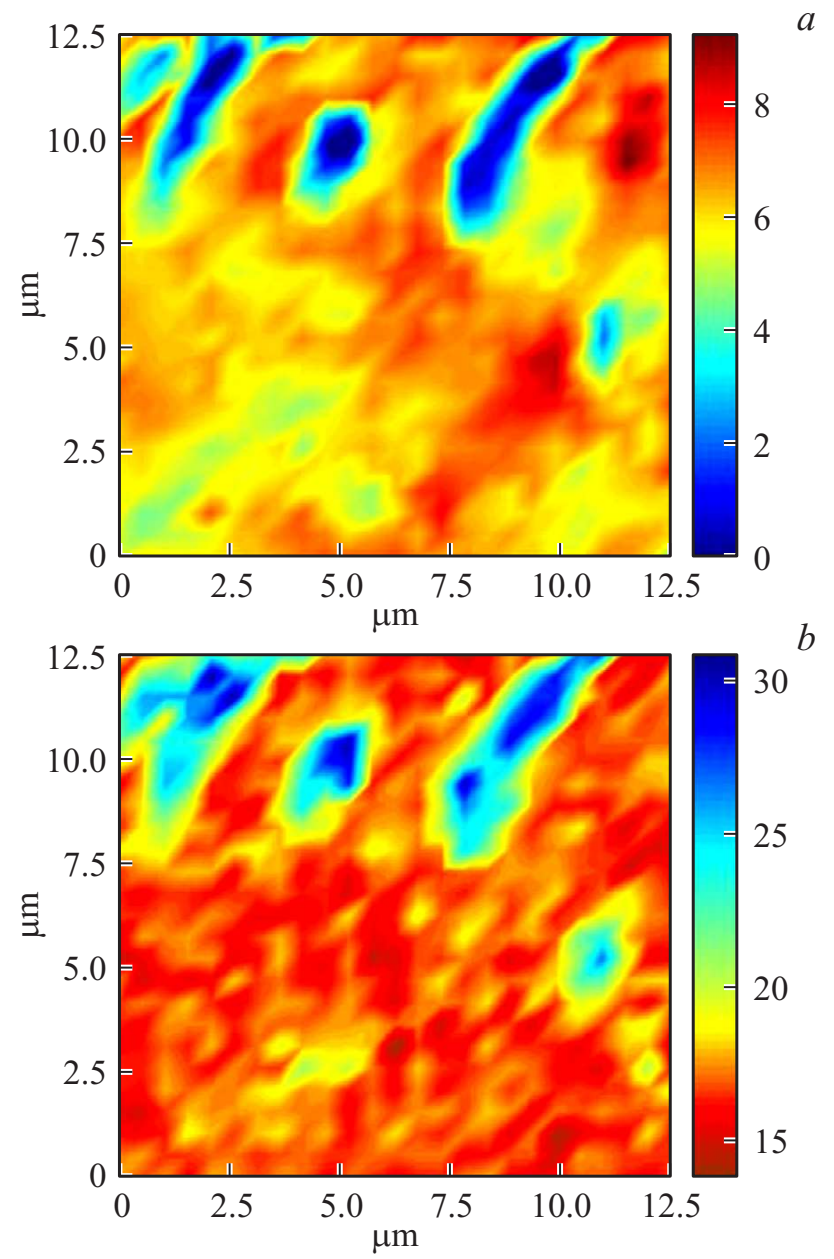

Рис. 5. Карта КРС сдвига линии $G(a)$ и карта значений ПШПВ линии $G(b)$ для образца после его прогрева при температуре $500^{\circ} \mathrm{C}$ в сверхвысоком вакууме.

в спектре. Таким образом, наблюдаемый на рис. 3 дополнительный высокочастотный сдвиг $G$ линии при практически неизменном положении $2 D$ линии следует связать с наличием избытка поверхностного заряда, созданного электронами.

Дополнительное подтверждение этому заключению следует из данных, представленных на рис. 4 и рис. 5. На рис. 4, $a$ и $b$ приведены гистограммы величин ПШПВ линии $G$, измеренные до и после проведения РФЭС-измерений. Хорошо видно, что этот параметр становится существенно меньше после прогрева образца в сверхвысоком вакууме при $500^{\circ} \mathrm{C}$. На рис. $5, a$ представлена карта сдвигов $\Delta \omega_{G}=\left(\omega_{G}-1601\right) \mathrm{cm}^{-1}$, а на рис. $5, b$ приведена соответствующая карта величин ПШШВ $\left(\right.$ в $\left.\mathrm{cm}^{-1}\right)$ линии $G$. Хорошо видна корреляция между двумя картами, а именно: чем больше величина $\Delta \omega_{G}$, тем меньше величина ПШПВ линии $G$.

В работах $[11,12]$ было показано, что появление избытка заряда любого знака в графене сопровождается увеличением времени жизни оптического фонона $E_{2 g}$, т.е уменьшением ПШПВ линии $G$ вследствие блокирования каналов распада фононов на электрондырочные пары. Величина ПШПВ линии $G$ достигает минимума, когда легирование вызывает сдвиг уровня Ферми, больший по величине, чем половина энергии оптического фонона $\left|E_{\mathrm{F}}\right|>\hbar \omega_{G} / 2$. Максимальный высокочастотный сдвиг линии $G$ составляет $\sim 7 \mathrm{~cm}^{-1}$. Согласно данным работы [12], эта величина должна соответствовать сдвигу уровня Ферми на 350-370 мэВ, что существенно превышает половину энергии оптического фонона $E_{2 g} \sim 100$ мэВ. Таким образом, наблюдаемый на рис. 4 дополнительный высокочастотный сдвиг линии $G$ и изменения величин ее ПШПВ следует приписать избытку поверхностного заряда. Вариации этого сдвига указывают на существование локальной неоднородности зарядовой плотности электронов в исследуемой области образца, средняя величина которой может быть оценена как $7 \cdot 10^{12} \mathrm{~cm}^{-2}[12]$.

\section{2. Данные АСM}

На рис. 6, $a$ и $b$ представлены карты топографии поверхности и распределения поверхностного потенциала области сканирования $10 \times 10$ мкм, а также их профили вдоль линий, отмеченных пунктиром. На изображении топографии поверхности различима серия вытянутых террас шириной $\sim 0.5$ мкм. Средняя высота террас (рис. 6, $d$ ) составляет $\sim 1$ нм (величина среднеквадратичного отклонения $\mathrm{rms}=0.6 \mathrm{Hм}$ ), что говорит об относительной гладкости исследуемой поверхности. На изображении распределения поверхностного потенциала (рис. $6, b)$ присутствуют светлые области (области повышенного потенциала) в виде вытянутых полос, направление которых совпадает с направлением террас на изображении топографии. Площадь светлых областей не превышает 10\% от площади изображения. Было установлено, что разность потенциалов между светлыми и темными областями составляет $\sim 140 \mathrm{MB}$. Такое значение соответствует разности поверхностных потенциалов между одно- и двухслойным графеном $[15,16]$. При этом светлые области на рис. $6, b$ следует отнести к двухслойному графену. Это заключение согласуется с результатами КРС-исследований, полученными на той же самой площади образца (рис. 6,c). Действительно, сравнение карт КРС (рис. 6,c) и распределения поверхностного потенциала (рис. 6,b), а также их соответствующих профилей (рис. $6, f$ и $e$ ), позволяет утверждать, что в светлых областях интенсивность линии $G$ больше. Следовательно, в них больше толщина графенового слоя.

\section{3. Данные ДМЭ, РФЭС, ФЭСУР и синхротронных измерений}

Картина ДМЭ, показанная на рис. 7, $a$, демонстрирует характерную для образца графен/ $\mathrm{SiC}(0001)$ структуру $(6 \sqrt{3} \times 6 \sqrt{3}) R 30^{\circ}$, возникающую вследствие несовпадения периодов решетки графена и поверхности $\mathrm{SiC}(0001)$ [17]. Другие упорядоченные фазы в картине 

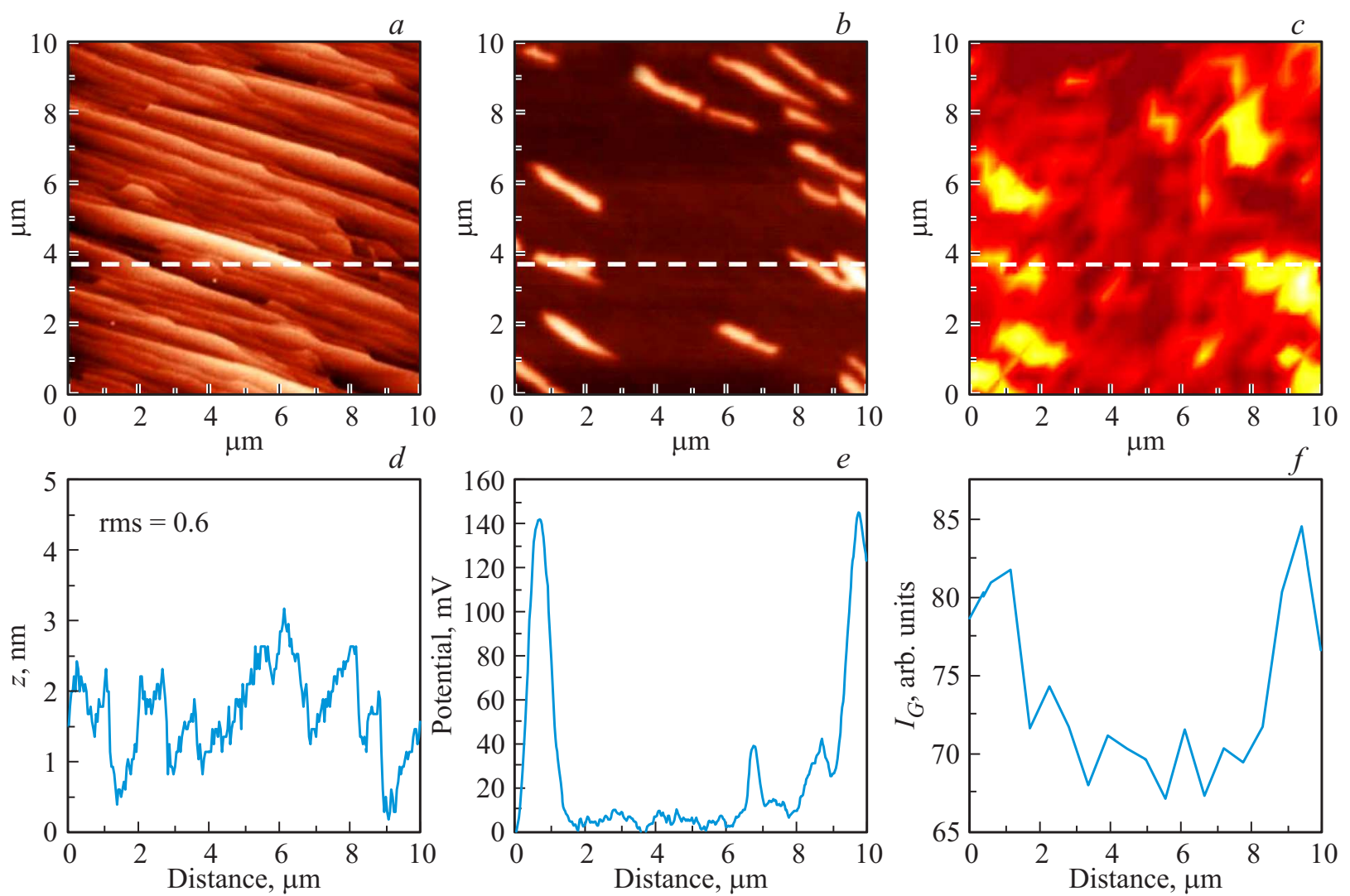

Рис. 6. Топография поверхности $(a)$ и ее профиль $(d)$, полученный вдоль линии, отмеченной пунктиром. Распределение поверхностного потенциала $(b)$ и соответствующий профиль $(e)$. Карта КРС интенсивности линии $G(c)$ и соответствующий профиль $(f)$.

ДМЭ не обнаружены. Четкие рефлексы свидетельствуют о строгой пространственной ориентации решетки графена. Для определения химического состава приповерхностной области был измерен обзорный спектр РФЭС (рис. $7, b)$ после удаления адсорбированных на воздухе молекул путем прогрева в сверхвысоком вакууме при температуре $500^{\circ} \mathrm{C}$. Помимо линий углерода и кремния, а также пиков характеристических потерь энергии электронов, в спектре обнаружено лишь небольшое количество кислорода. Его концентрация в верхних атомных слоях $\mathrm{SiC}$ составляет примерно 1 ат.\%.

Детальный анализ формы спектров РФЭС в области линии $\mathrm{C} 1 s$ дает возможность оценить среднюю толщину графена. Такую оценку нетрудно провести, если пренебречь упругим рассеянием фотоэлектронов, эффектами фотоэлектронной дифракции, а также процессами shakeup при фотоэмиссии из уровня $1 s$. При этих допущениях интенсивность фотоэмиссии из выбранного атомного слоя пропорциональна концентрации атомов в слое и коэффициенту ослабления интенсивности в результате неупругого рассеяния в вышележащем слое вещества. Этот коэффициент при эмиссии по нормали к поверхности равен $\exp \left(-d / \lambda\left(E_{\mathrm{k}}\right)\right)$, где $d-$ толщина вышележащего слоя, а $\lambda\left(E_{\mathrm{k}}\right)$ - длина свободного пробега электрона по отношению к неупругому рассеянию в этом слое при кинетической энергии электрона $E_{\mathrm{k}}$.

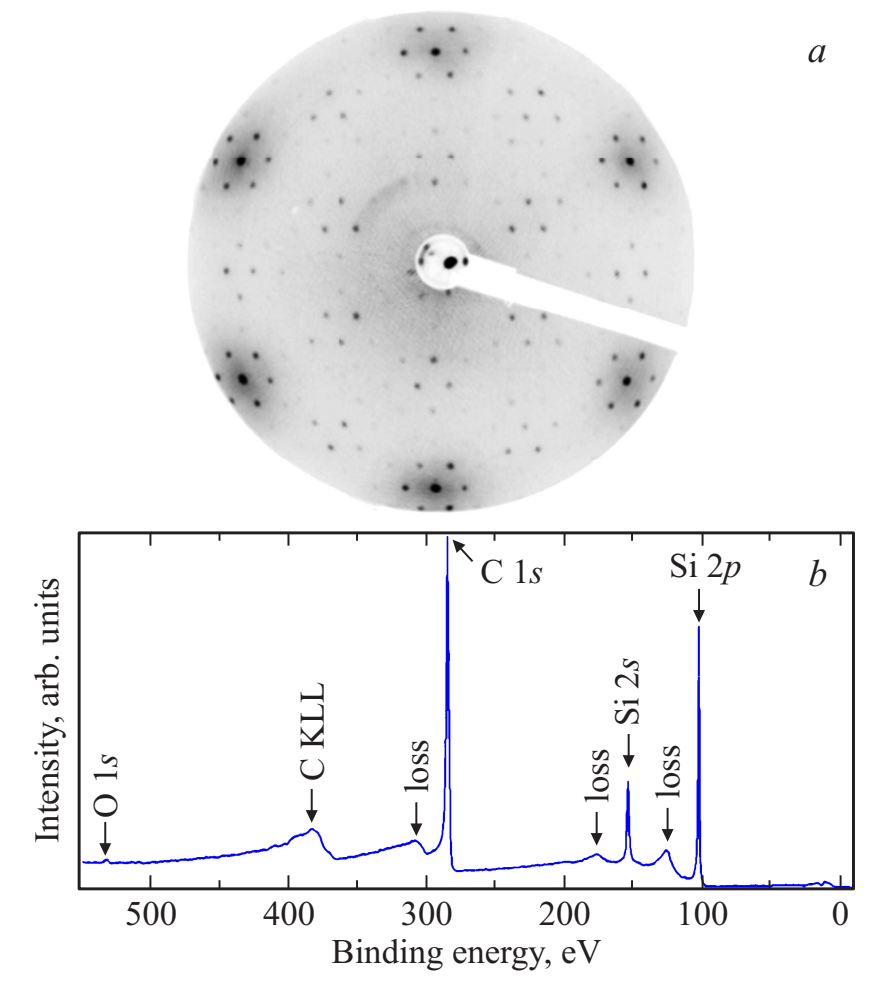

Рис. 7. Картина дифракции медленных электронов. Энергия электронов 90 эВ $(a)$. Обзорный спектр РФЭС при энергии фотонов 650 эВ $(b)$. 

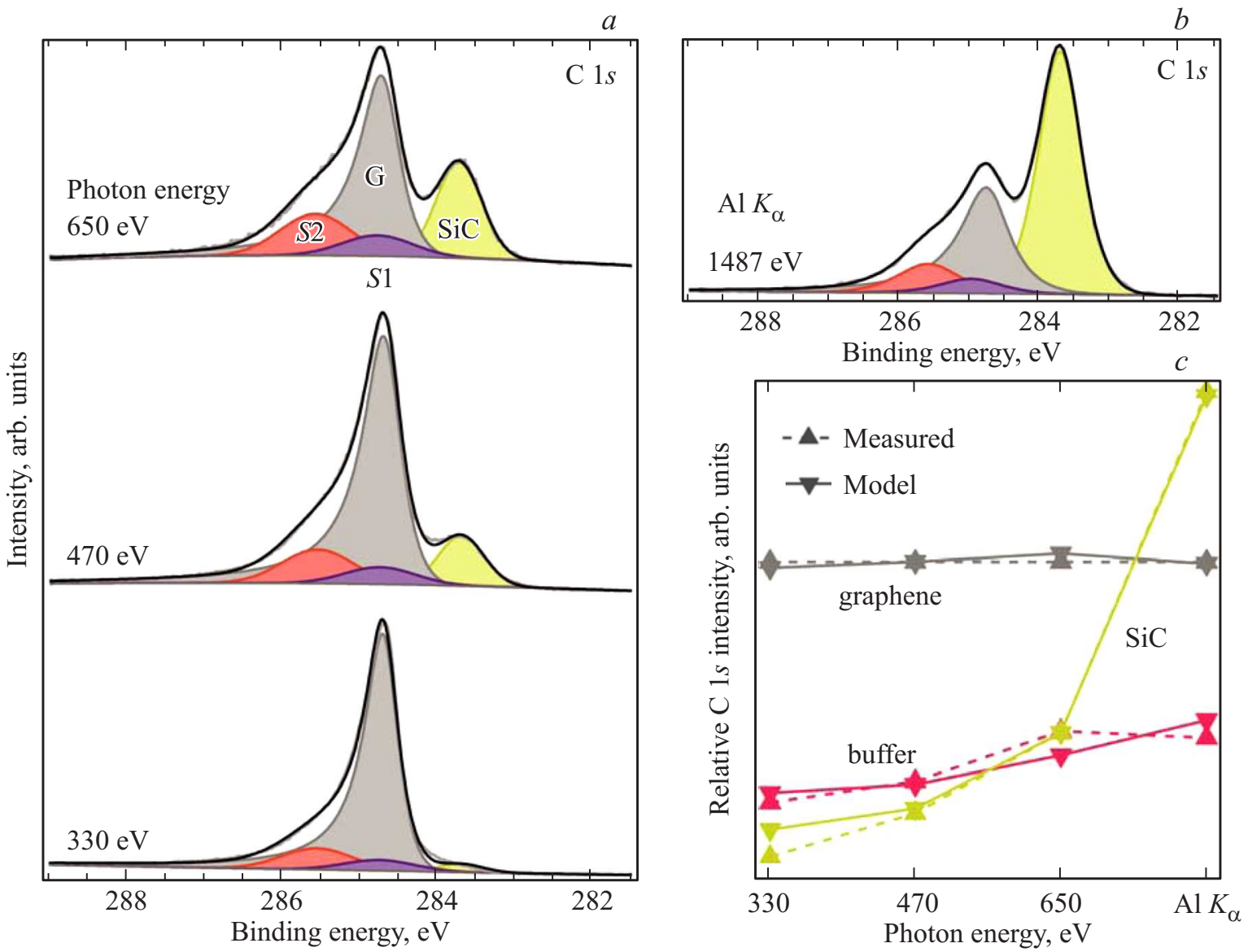

Рис. 8. Спектры РФЭС, измеренные в области $\mathrm{C} 1 s$ при различных энергиях фотонов $(a, b)$. Результаты моделирования интенсивности отдельных спектральных компонент $(c)$.
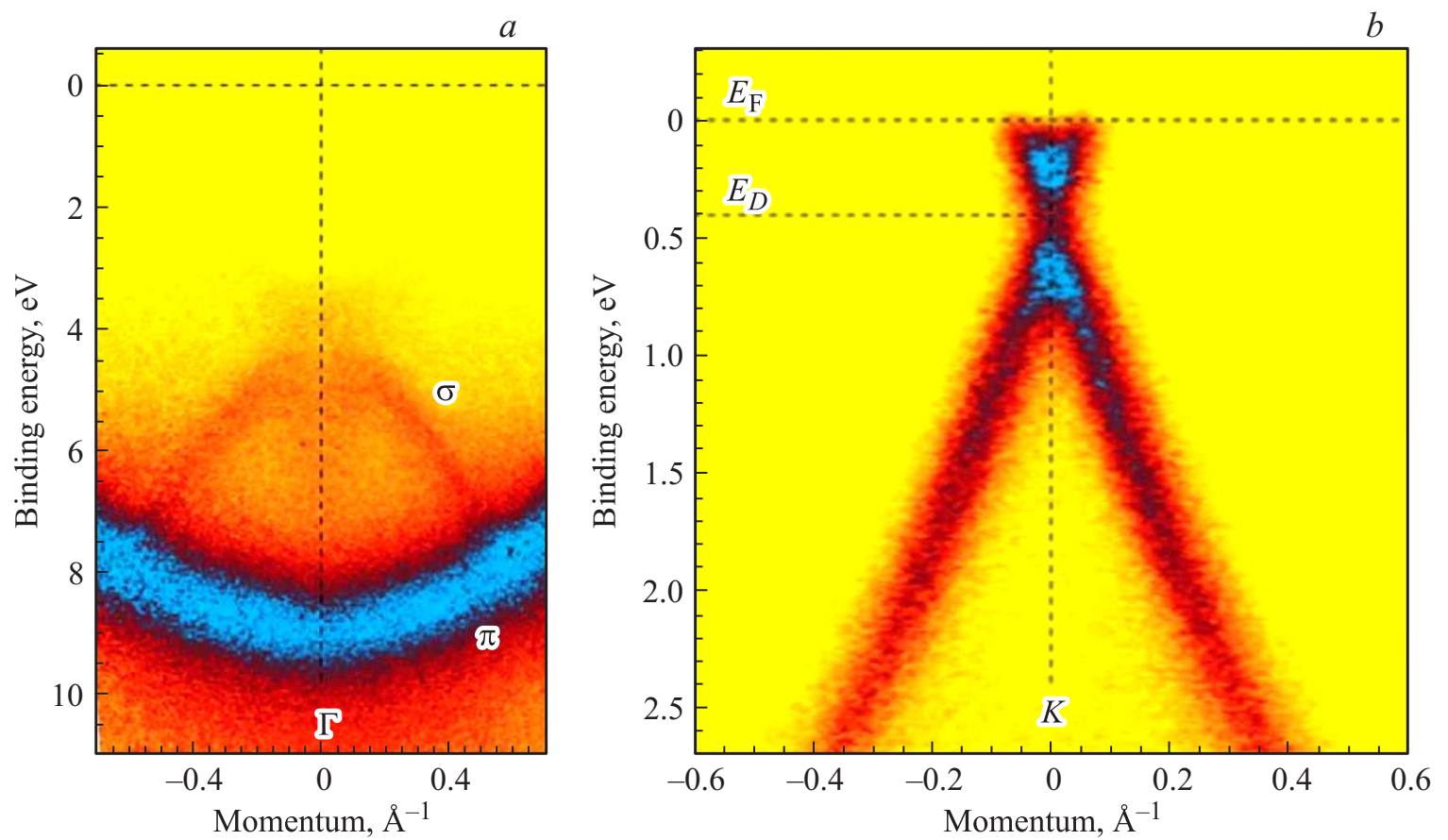

Рис. 9. Структура электронной валентной зоны в окрестности точек $Г(a)$ и $K(b)$ зоны Бриллюэна. Данные ФэСуР получены с использованием излучения Не II (40.8 эВ). 
Форму спектра $\mathrm{C} 1 s$ можно описать четырьмя компонентами, показанными на рис. 8, $a$. Известно, что правая компонента соответствует углероду в $\mathrm{SiC}$ и имеет энергию связи около 283.7 эВ [17]. Буферный слой углерода, имеющий графеноподобную структуру, характеризуется двумя компонентами $S 1$ и $S 2$ при энергиях связи 285.0 и 285.6 эВ соответственно. Различная энергия этих компонент связана с тем, что примерно треть атомов углерода образует сильную ковалентную связь с кремнием. Поскольку пик $S 1$ не различим на фоне графенового пика $G$, имеющего энергию около 284.7 эВ, то при разложении спектра на компоненты необходимо учитывать, что соотношение интенсивностей $S 1: S 2$ составляет примерно $1: 2$. Следует также отметить, что компоненты $S 1, S 2$ и $\mathrm{SiC}$ достаточно хорошо аппроксимируются симметричными пиками с формой близкой к гауссовой, тогда как пик графена $G$ имеет ярко выраженную асимметрию и может быть аппроксимирован, например, асимметричным произведением контуров Гаусса и Лоренца [18].

Для надежного определения толщины графена проводился анализ спектров РФЭС, полученных при четырех энергиях фотонов, обеспечивающих различную глубину анализа в диапазоне 5-23 А. Толщину слоев определяли путем подбора значений толщины графена и буферного слоя, обеспечивающих наилучшее совпадение рассчитанных и измеренных интенсивностей отдельных компонент спектров $\mathrm{C} 1 s$.

Результаты приведены на рис. $8, c$, где показаны относительные интенсивности для оптимальных значений толщины: $3.3 \AA$ для буферного слоя и $5.5 \AA$ для графена. Это соответствует количеству $s p^{2}$-слоев углерода 1.0 и 1.6 для буферного слоя и графена соответственно.

Известно, что в зависимости от числа слоев графена изменяется электронная структура валентной зоны. Для однослойного графена характерен дираковский конус электронных состояний в точке $K$ зоны Бриллюэна, тогда как формирование второго слоя приводит к энергетическому расщеплению конуса и удвоению числа состояний [17]. Данные ФЭСУР по электронной структуре валентной зоны системы графен/SiC $(0001)$ показаны на рис. 9. В центре зоны Бриллюэна наблюдаются характерные для графена $\sigma$-состояния, а также $\pi$-состояния с параболической дисперсией. В точке $K$ виден нерасщепленный дираковский конус. Это свидетельствует о том, что на поверхности доминирует однослойное графеновое покрытие. Такое заключение согласуется с результатами КРС, АСМ и РФЭС о характере роста образца, как преимущественно однослойного графена с небольшим количеством $(\sim 10 \%)$ включений двухслойных островков с субмикронными размерами. Из данных ФЭСУР также видно, что точка Дирака находится ниже уровня Ферми и имеет энергию связи $E \approx 0.4$ эВ. Это однозначно указывает на $n$-тип допирования с концентрацией электронов в зоне проводимости $n=E^{2} / \pi\left(\hbar v_{\mathrm{F}}\right)^{2} \approx 10^{13} \mathrm{~cm}^{-2}$. Эта величина согласуется с данными КРС, демонстрирующими значительный перенос заряда в образце после отжига в вакууме. Для

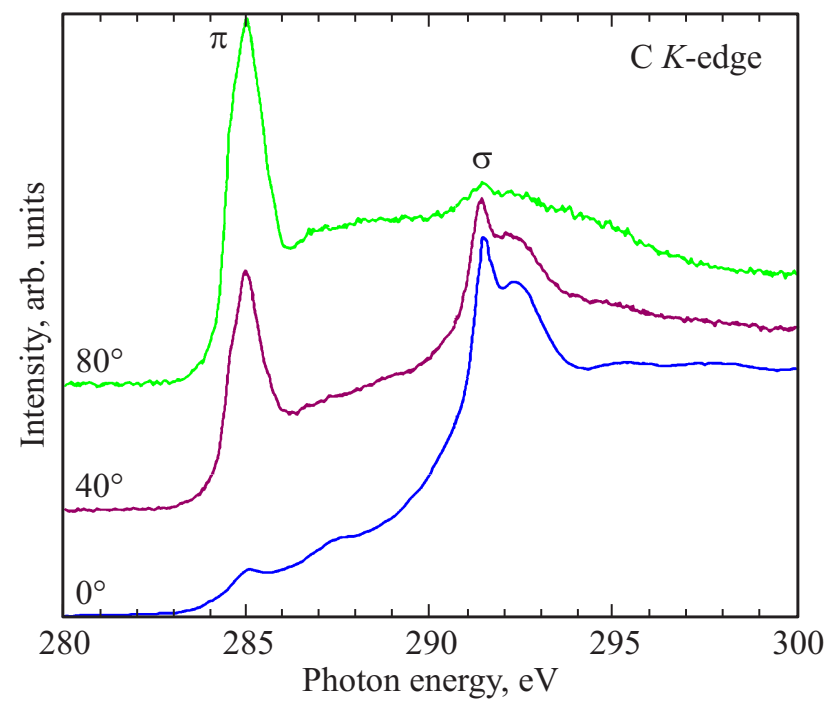

Рис. 10. Ближняя тонкая структура рентгеновских спектров поглощения (NEXAFS). Над спектрами указан угол между нормалью к поверхности образца и вектором линейной поляризации фотонов.

выяснения причин слабого переноса заряда в образцах до отжига в вакууме, мы планируем в дальнейшем систематически изучить влияние внешних условий на зарядовое состояние графена.

Убедительную демонстрацию того, что и графен и буферный слой представляют собой планарные $s p^{2}$ структуры, дает спектроскопия поглощения вблизи $K$-края углерода (NEXAFS). На рис. 10 показаны спектры NEXAFS, записанные в режиме частичного квантового выхода. При измерениях использовалась задерживающая сетка с потенциалом $-200 \mathrm{~B}$, отсекающая медленные электроны, выходящие преимущественно из объема $\mathrm{SiC}$. Это обеспечило высокую поверхностную чувствительность спектров. Полученные данные представляют собой поток фотоэлектронов с энергией свыше 200 эВ, возбужденных в верхних атомных слоях образца, поэтому спектры характеризуют преимущественно слои графена и буфера. Характерной особенностью спектров поглощения графеноподобных структур является наличие $\pi$ и $\sigma$-резонансов, имеющих противоположные угловые зависимости. Максимальная интенсивность $\sigma$-резонанса ожидается, когда вектор поляризации излучения лежит в плоскости графена (угол $0^{\circ}$ ). При этом интенсивность $\pi$-резонанса должна стремиться к нулю. Такая зависимость наблюдается на рис. 10 и свидетельствует о том, что графен и буферный слой являются планарными $s p^{2}$ структурами, ориентированными параллельно поверхности $\mathrm{SiC}$. Однако интенсивность в области $\pi$-резонанса не полностью пропадает при угле $0^{\circ}$. Это объясняется ненулевым вкладом в спектры от края поглощения $\mathrm{SiC}$, а также может указывать на небольшую корругацию $s p^{2}$ слоев в результате взаимодействия с подложкой. 


\section{4. Заключение}

С использованием результатов комплексных исследований установлена связь структурных, химических и электронных характеристик графеновых пленок, выращенных методом термического разложения поверхности $\mathrm{SiC}$, с технологическими режимами роста. Это позволило осуществить оптимизацию технологических параметров и создать воспроизводимую технологию роста однослойной пленки графена с небольшой долей мелких включений двухслойного графена на Si-грани подложки $6 H$-SiC. Установлено, что наилучшими структурными характеристиками обладают пленки графена, выращенные с использованием следующих технологических параметров: температура роста $1850^{\circ} \mathrm{C}$, время роста 10 мин, давление аргона в ростовой камере 750 Торр, скорость нагрева образца $100-150^{\circ} \mathrm{C} /$ мин. В целом, образцы можно охарактеризовать как высококачественный графен, что предполагает возможность создания приборов на их основе.

Д.Ю.У. благодарит DFG-СПбГУ за поддержку в рамках проекта 11.65.42.2017, а также РФФИ (проект 17-02-00427). Авторы благодарны Helmholtz Zentrum Berlin für Materialien und Energie за поддержку в рамках Российско-Германской лаборатории.

\section{Список литературы}

[1] A.C. Ferrari, F. Bonaccorso, V. Fal'ko, K.S. Novoselov et al. Nanoscale, 7, 4598 (2015).

[2] K.V. Emtsev, A. Bostwick, K. Horn, J. Jobst, G.L. Kellogg, L. Ley, J.L. McChesney, T. Ohta, S.A. Reshanov, J. Rohrl, E. Rotenberg, A.K. Schmid, D. Waldmann, H.B. Weber, T. Seyller. Nature Materials, 8, 203(2009).

[3] G.R. Yazdi, T. Iakimov, R. Yakimova. Crystals, 6, 53 (2016).

[4] J.L. Tedesco, B. VanMil, R. Myers-Ward, J.M. McCrate, S.A. Kitt, P.M. Campbell, G.G. Jernigan, J.C. Culbertson, C.R. Eddy jr, D.K. Gaskill. Appl. Phys. Lett., 95, 122102 (2009).

[5] Н.В. Агринская, В.А. Березовец, В.И. Козуб, И.С. Котоусова, А.А. Лебедев, С.П. Лебедев, А.А. Ситникова. ФТП, 47, 267 (2013).

[6] А.А. Лебедев, С.П. Лебедев, С.Н. Новиков, В.Ю. Давыдов, А.Н. Смирнов, Д.П. Литвин, Ю.Н. Макаров, В.С. Левицкий. ЖТФ, 86, 135 (2016).

[7] A.C. Ferrari, D.M. Basko. Nature Nanotech., 8, 235 (2013).

[8] R. Beams, L.G. Cancado, L. Novotny. J. Phys.: Condens. Matter, 27, 083002 (2015).

[9] L.G. Cançado, K. Takai, T. Enoki, M. Endo, Y.A. Kim, H. Mizusaki, A. Jorio, L.N. Coelho, R. Magalhães-Paniago, M.A. Pimenta. Appl. Phys. Lett., 88, 163106 (2006).

[10] Z.H. Ni, W. Chen, X.F. Fan, J.L. Kuo, T. Yu, A.T.S. Wee, Z.X. Shen. Phys. Rev. B, 77, 115416 (2008).

[11] J. Yan, Y. Zhang, P. Kim, A. Pinczuk. Phys. Rev. Lett., 98, 166802 (2007).

[12] A. Das, S. Pisana, B. Chakraborty, S. Piscanec, S.K. Saha, U.V. Waghmare, K.S. Novoselov, H.R. Krishnamurthy, A.K. Geim, A.C. Ferrari, A.K. Sood. Nature Nanotech., 3, 210 (2008)
[13] J.E. Lee, G. Ahn, J. Shim, Y.S. Lee, S. Ryu. Nature Commun., 3, 1024 (2012).

[14] F. Ding, H. Ji, Y. Chen, A. Herklotz, K. Dörr, Y. Mei, A. Rastelli, O.G. Schmidt. Nano Lett., 10, 3453 (2010).

[15] V. Panchal, R. Pearce, R. Yakimova, A. Tzalenchuk, O. Kazakova. Sci. Rep., 3, 2597 (2013).

[16] T. Filleter, K.V. Emtsev, Th. Seyller, R. Bennewitz. Appl. Phys. Lett., 93, 133117 (2008).

[17] C. Riedl, C. Coletti, U. Starke. J. Phys. D: Appl. Phys., 43, 374009 (2010).

[18] R. Hesse, T. Chassé, R. Szargan. Fresenius J. Anal. Chem., 365, 48 (1999).

Редактор А.Н. Смирнов

\section{Study of crystal and electronic structure of graphene films grown on $6 \mathrm{H}$-SiC (0001)}

V.Yu. Davydov 1,3, D.Yu. Usachov ${ }^{2}$, S.P. Lebedev', A.N. Smirnov ${ }^{\mathbf{1}, 3}$, V.S. Levitskii ${ }^{1}$, I.A. Eliseyev ${ }^{\mathbf{1 , 2}}$, P.A. Alekseev' ${ }^{1}$, M.S. Dunaevskiy ${ }^{1}$, O.Yu. Vilkov' ${ }^{2}$, A.G. Rybkin ${ }^{2}$, A.A. Lebedev ${ }^{1}$

${ }^{1}$ loffe Institute,

194021 St. Petersburg, Russia

2 Saint Petersburg State University,

199034 St. Petersburg, Russia

3 ITMO University,

197101 St. Petersburg, Russia

Abstract Structural, chemical and electronic properties of epitaxial graphene films grown by thermal decomposition of the Si-face of semi-insulating $6 \mathrm{H}-\mathrm{SiC}$ in an argon environment is studied by Raman spectroscopy, atomic force microscopy, electron diffraction, $X$-ray photoelectron spectroscopy, angle-resolved photoemission spectroscopy and $X$-ray absorption spectroscopy at the carbon $K$-edge. It is shown that the results of systematic complex investigations allowed optimization of growth parameters and creation of a reliable growth technology for high-quality monolayer graphene films with a small fraction of bilayer graphene inclusions. 